\title{
IMPUESTO NEGATIVO AL INGRESO DEL TRABAJO: UNA POLÍTICA CONTRA LA DESIGUALDAD
}

\author{
Andrés Hernando \\ Centro de Estudios Públicos \\ Estéfano Rubio \\ Centro de Estudios Públicos
}

\begin{abstract}
Resumen: Este artículo presenta el impuesto negativo al ingreso del trabajo (INIT), o crédito impositivo, un instrumento que consiste en complementar con transferencias en dinero los ingresos de los trabajadores de menores remuneraciones. Como política social, tiene la ventaja de ser un mecanismo eficaz para atacar la desigualdad, al tiempo que no distorsiona de forma importante la decisión ociotrabajo, promueve la inserción laboral y su formalización, disminuye la pobreza y reduce el estigma asociado a ser beneficiario de programas sociales. Se ofrece este instrumento con dos fines: (i) como una política para efectivamente ser aplicada y (ii) como un punto de referencia contra el cual contrastar qué tan costo-efectiva puede ser cualquier otra política pública que apunte a reducir la desigualdad. El trabajo aplica un algoritmo genético de optimización numérica,
\end{abstract}

Andrés Hernando. PhD en economía por la Harvard University e ingeniero civil en computación y máster por la Universidad de Chile. Profesor en la Universidad Adolfo Ibáñez (UAI). Investigador del Centro de Estudios Públicos (CEP). Email: ahernando@cepchile.cl.

EstéFAno Rubio. Economista y máster en economía por la Universidad de Chile. Investigador del CEP. Email: erubio@cepchile.cl.

Los autores agradecen los comentarios y sugerencias de Harald Beyer, Slaven Razmilic y dos árbitros anónimos que contribuyeron en forma importante a dar forma a este artículo. Como siempre, cualquier error u omisión en el texto final es responsabilidad exclusiva de los autores. 
que permite ir evaluando los parámetros óptimos que minimizan la desigualdad de ingresos, y muestra que, por ejemplo, con un aporte de 5.000 millones de dólares anuales, el Gini caería entre 4,7 y 6,1 puntos; una reducción mayor a toda la observada entre 1990 y 2015. PALABras Clave: desigualdad, empleo, subsidio, políticas sociales, benchmark de desigualdad.

RECIBIDO: mayo 2016; ACEPTADO: mayo 2017.

JEL: D31, H24, I38.

\section{EARNED INCOME TAX CREDIT: A PUBLIC POLICY AGAINST INEQUALITY}

ABSTRACT: This article presents the earned income tax credit (EITC), an instrument that consists in topping up lower-paid workers' earnings with cash transfers. As a social policy, it has the advantage of being an effective mechanism for tackling inequality that does not greatly interfere with the trade-off between leisure and work but encourages labour market participation and formalization, cuts poverty and reduces the stigma attached to being a social programme beneficiary. This instrument is offered for two purposes: (i) as an actual policy option and (ii) as a benchmark for contrasting the potential cost-effectiveness of any other public policy intended to reduce inequality. The paper applies a genetic algorithm for numerical optimization to evaluate the parameters that optimally minimize income inequality and shows that, for example, spending 5.000 million dollars a year would reduce the Gini by between 4.7 and 6.1 points, which is more than the entire reduction recorded between 1990 and 2015.

Keywords: inequality, employment, entitlements, social policies, inequality benchmark.

ReCEIVED: May 2016; ACCEPTED: May 2017.

JEL: D31, H24, I38.

\section{INTRODUCCIÓN}

no de los mayores desafíos pendientes de la sociedad chilena es reducir los altos índices de desigualdad que presenta. A pesar de la prosperidad económica y la reducción de la pobreza que ha evidenciado el país durante los últimos 25 años, la desigualdad de ingresos se ha mantenido elevada. Los datos muestran una caída en el índice de 
Gini ${ }^{1}$ para los ingresos autónomos per cápita desde 0,53 en 1990 a 0,50 en $2013 .^{2}$

Desde hace algún tiempo, la ciudadanía parece estar más consciente de estas desigualdades, lo cual ha redundado en mayores y más sostenidas demandas a los gobiernos por disminuir estas cifras. El desarrollo de una sociedad más empoderada y demandante ha llevado a la constante búsqueda de mecanismos para alcanzar este fin de mayor equidad en la distribución de los logros económicos del país. Se presentan, así, propuestas de política pública que se instalan en el debate público y se dotan de respaldo ciudadano y de amplio capital político que pudieran no ser las técnicamente óptimas para alcanzar esta meta.

Debido a lo anterior, se hace indispensable contar con una herramienta de política pública que permita medir o — mejor dicho, en conformidad a nuestros fines - contrastar la efectividad de una propuesta de política cuyo fin sea reducir la desigualdad, con un escenario óptimo de minimización de esta desigualdad de ingresos. Eso es lo que presentará este trabajo. A continuación, introducimos un impuesto negativo al ingreso del trabajo (INIT), ${ }^{3}$ que permite maximizar la disminución en el índice de Gini para cualquier nivel de presupuesto que se desee gastar.

El INIT se muestra como un mecanismo eficaz de política pública para atacar la desigualdad. Por un lado, desde un punto de vista económico, es técnicamente apropiado porque no distorsiona de forma importante la decisión ocio-trabajo, promueve la inserción laboral y fomenta la formalización. ${ }^{4}$ Por el otro lado, y no menos importante, el INIT también puede considerarse como una política con amplio potencial político para su respaldo, ya que incentiva el trabajo de los más pobres, disminuye la pobreza y reduce el estigma asociado a ser beneficiario de programas sociales.

${ }^{1}$ El coeficiente de Gini, que es la medida más utilizada para medir desigualdad, toma valores entre 0 (máxima igualdad) y 1 (máxima desigualdad).

${ }^{2}$ Cifras calculadas a partir de la encuesta Casen. Debido a que, a lo largo de la serie, ha cambiado la forma en que se calcula el índice, las cifras corresponden a nuestros cálculos. Ellas incluyen las observaciones con ingresos nulos y consideran los ingresos corregidos por no respuesta, pero sin el ajuste por cuentas nacionales que realizaba la Cepal entre 1990 y 2011.

${ }^{3}$ El cual corresponde a un esquema de EITC (earned income tax credit).

${ }^{4}$ Considerando que los ingresos bonificados son aquellos reportados al sistema impositivo. 
Sin embargo, para poder hablar de optimalidad en la reducción de la desigualdad, requerimos que nuestra herramienta sea, además de eficaz, eficiente. Por esta razón es que el diseño del INIT es fundamental. Para determinar los parámetros respectivos, desarrollamos un proceso que consta de una simulación de cinco pasos: (1) determinar un ingreso imponible; (2) calcular un salario esperado y una probabilidad de trabajar; (3) definir quiénes calificarían para el INIT y el monto que cobrarían; (4) establecer nuevas incorporaciones al mercado laboral; y, finalmente, (5) calcular el efecto en desigualdad. Todo esto permitirá que un algoritmo genético ${ }^{5}$ de optimización numérica nos deje ir evaluando los parámetros óptimos que puedan minimizar la desigualdad de ingresos, empleando para ello el ya descrito método de simulación.

Nuestro método encuentra que, con un presupuesto de $5 \mathrm{mil} \mathrm{mi-}$ llones de dólares, el INIT disminuiría la desigualdad, medida al usar el coeficiente de Gini — entre 4,7 y 6,1 puntos—- que es una caída mayor a la lograda por la economía chilena en todo el periodo entre 1990 y 2015. Además, abarcaría entre 1.600 .000 y 2.500 .000 potenciales beneficiarios directos.

Es importante mencionar que gran parte de los países de la OCDE consiguen disminuir considerablemente sus índices de desigualdad usando impuestos y transferencias y, sólo en menor medida, mediante prestaciones de servicios. En la tabla 1 podemos apreciar cómo cambia el coeficiente de Gini para Chile y los países de la OECD comparando (1) el ingreso antes de impuestos y transferencias; (2) luego, de impuestos y transferencias; y (3), finalmente, lo que resulta de agregar el monto correspondiente a servicios sociales (como educación, salud, pensiones, entre otros). De aquí podemos concluir que Chile tiene mucho que mejorar en sus sistemas de transferencias a las familias. Es también en línea con esta perspectiva que planteamos una política como el INIT.

Posterior a esta introducción, la sección 2 presenta los fundamentos teóricos detrás de un el INIT basado en un modelo de earned income tax credit (EITC); la sección 3, en tanto, desarrolla en detalle los métodos de simulación y optimización; la sección 4 expone los resultados de los distintos casos analizados; mientras que la sección 5 ofrece una conclusión.

\footnotetext{
${ }^{5}$ Éste se explicará detalladamente en el apartado de optimización.
} 
Tabla 1. IMPACTO DISTRIBUTIVO DE IMPUESTOS Y TRANSFERENCIAS Y SERVICIOS SOCIALES: ÍNDICE GINI CHILE VS OECD (2011)

\begin{tabular}{lcc}
\hline & Chile & OECD \\
\hline Ingreso antes de impuestos y transferencias & 53,1 & 41,0 \\
Ingreso disponible (después de impuesto a la renta & 50,0 & 29,1 \\
y transferencias en dinero) & & \\
Ingreso ampliado (disponible + servicios sociales) & 41,1 & 23,5 \\
\hline
\end{tabular}

Fuente: Larrañaga y Rodriguez (2014).

\section{EL EITC Y SU RACIONALIDAD ECONÓMICA}

En países de Europa, América del Norte y Oceanía se ha puesto énfasis en el sistema tributario como mecanismo no sólo para recaudar, sino que también para llevar a cabo políticas de redistribución. Destaca, entre éstas, la entrega de beneficios sociales en forma de un crédito en la devolución anual de impuestos para grupos de bajos ingresos. En Estados Unidos se originó un sistema de crédito tributario reembolsable que se focaliza en las familias de más bajos ingresos, denominado earned income tax credit (en adelante EITC). La entrega de éste está sujeta a que el individuo trabaje y, si el monto del crédito es mayor de lo que una familia debe pagar en impuestos, entonces se entrega la diferencia a través de una transferencia monetaria. En nuestro caso, establecimos como condición la entrega del beneficio a personas que estuviesen en el tramo exento de impuesto a la renta, de tal forma que en la práctica equivaldría a un impuesto inverso o negativo.

Tal como señalan Agostini et al. (2013), el crédito tributario al ingreso tiene ventajas como mecanismo de redistribución por cuanto: (i) reduce la pobreza, (ii) incentiva el trabajo de los más pobres y (iii) disminuye el estigma asociado a ser beneficiario de programas sociales. Otras ventajas de esta política son que, (iv) al igual que las transferencias condicionadas, ${ }^{6}$ puede ser más aceptable políticamente al exigir

${ }^{6}$ Es importante señalar que aunque los impuestos negativos al ingreso se pagan sólo a quienes generan ingresos formales, éstos no entran en la lógica de las transferencias condicionales de efectivo (conocidas por su sigla en inglés, CCT); esto, por cuanto una CCT está condicionada en acciones que (i) apuntan a aumentar el stock de largo plazo de capital humano en un grupo de la población que sistemáticamente subinvierte en él; y (ii) una CCT se condiciona en acciones que todas las familias pueden cumplir si optan por hacerlo. Es obvio que la participación en el empleo formal no satisface ninguna de estas características (Lindert, Skaufios y Shapiro 2006). 
un esfuerzo por parte del beneficiario, algo que no demandan las transferencias no condicionadas (Fizbein et al. 2009); (v) posee menores costos administrativos, ya que aprovecha la institucionalidad de administración tributaria ya existente en el país; y que si bien un programa como el EITC no cubre a trabajadores informales, en el largo plazo (vi) tenderá a hacer más atractiva la formalización de éstos.

De esta manera, el EITC es una política que permite incrementar no sólo los ingresos monetarios, sino que al mismo tiempo puede aumentar los ingresos autónomos de las familias. Esto último es una potencial consecuencia indirecta, producto de la creación de incentivos para que personas que se encontraban fuera del mercado laboral decidan incorporarse y generar una nueva fuente de ingresos para el hogar. Este punto es relevante, pues, según Beyer (2011), el talón de Aquiles de la política redistributiva en Chile es el empleo. Su trabajo concluye que existe una enorme heterogeneidad en el promedio de las tasas de ocupación en Chile, que alcanza, en el quinto quintil (el de mayores ingresos), al 67 por ciento, mientras que en el primer quintil (el de más bajos ingresos) llega solamente al 28 por ciento. Al mismo tiempo, diagnostica que existe una preocupante declinación en la participación laboral de los grupos de menor calificación. Por último, sugiere que una política redistributiva no puede renunciar a proveer empleos a la población menos calificada, puesto que éste es el instrumento probablemente más efectivo para avanzar en equidad. En línea con ello, esperamos que una política como el EITC contribuya a cumplir dichos objetivos.

La figura 1 presenta un gráfico de ocio-consumo ${ }^{7}$ típico. En ausencia del crédito tributario al ingreso, la restricción presupuestaria corresponde a la línea dada por $A E .^{8}$ Luego, el EITC incluye una primera fase (phase in), tramo $A B$, de subsidio al ingreso laboral proporcional al salario $\left(w\left(1+t_{1}\right)\right)$, para los ingresos más bajos. Otra (flat region), tramo

${ }^{7}$ Esta figura muestra cómo una persona puede "intercambiar" entre dos "bienes": consumo y ocio. Es decir, puede optar por tener más tiempo libre (trabajando menos) a cambio de reducir los recursos de los cuales puede consumir y viceversa. Nótese que en esta figura las horas de ocio se miden en el eje horizontal (abscisas), siendo crecientes de izquierda a derecha. Esto quiere decir que las horas de trabajo son crecientes en la dirección contraria, ya que la suma de las horas de trabajo y ocio es siempre constante e igual al número de horas disponibles en el día.

${ }^{8}$ Cuya pendiente corresponde al precio relativo del ocio, esto es, el salario real. 
$B C$, en la que el subsidio alcanza su tope máximo y se otorga un monto fijo; nótese que la pendiente de la restricción presupuestaria en este tramo es paralela a la que habría sin EITC (w). Y una tercera (phase out), tramo $C D$, en la que el crédito es inversamente proporcional al ingreso $\left(w\left(1+t_{2}\right)\right)$, decreciendo hasta desaparecer. El EITC hace que el ingreso sea siempre mayor o igual a la situación previa al programa; y es esto lo que permite disminuir la desigualdad y pobreza.

Por otra parte, este esquema de subsidio se caracteriza por conllevar una expansión no lineal de la restricción presupuestaria, de tal manera que el salario (precio relativo entre ocio y consumo) varíe acorde a la pendiente de esta última. Esto provoca que los incentivos generados sobre la oferta laboral cambien de acuerdo a cada tramo. Ésta es una de las ventajas del EITC con respecto a otros subsidios o beneficios sociales que solamente generan un alza del ingreso no laboral, lo que produce desplazamientos paralelos de la restricción presupuestaria, provocando que con más horas de ocio (y menos de trabajo) se puedan alcanzar iguales niveles de consumo que antes del beneficio.

Figura 1. EFECTO DEL EITC EN LA RESTRICCIÓN PRESUPUESTARIA

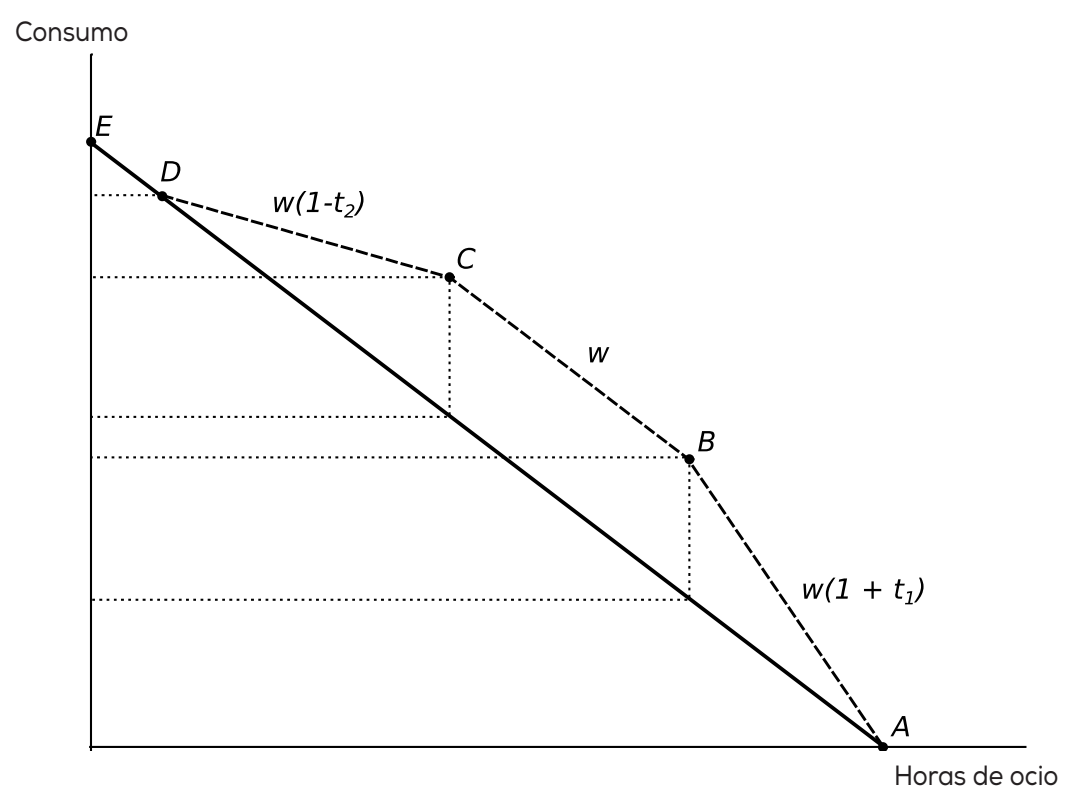

Fuente: Elaboración de los autores. 
En la figura 2 podemos apreciar los efectos sobre la oferta laboral que provoca el crédito impositivo al ingreso de acuerdo a cada tramo, donde la situación inicial sin EITC está representada por el punto $P$ y la situación con EITC está representada en el punto $R$. En el caso (a) vemos que si un individuo no trabajaba antes de la instauración de la política, cuando ésta se implementa siempre podría acceder a un nivel de bienestar mayor, generándose de esta forma incentivos al trabajo. Esto provoca un efecto positivo en participación laboral, por ejemplo, en mujeres y jóvenes, que suelen ser grupos con bajas tasas de ocupación; pero también es sumamente positivo para familias de bajos ingresos en las que nadie trabaja, ya que la inserción al mercado laboral generará un incremento significativo en los ingresos autónomos del hogar.

No obstante, para individuos que ya se encontraban trabajando antes de la política, estos enfrentan incentivos ambiguos con respecto a la determinación de las horas a trabajar. En el segundo tramo, (b), sólo se genera un efecto ingreso, el cual producirá incentivos a aumentar las horas de ocio (y disminuir las laborales). Por último, en el tercer segmento, (c), tanto el efecto sustitución como el efecto ingreso generan incentivos negativos en trabajo. Por otra parte, según Eissa y Liebman (1996), quienes estén cerca de ser elegibles pero que están por encima del tercer tramo puede que opten por disminuir sus horas laborales para ganar menos ingresos y así ser elegibles para el beneficio. Por lo tanto, en teoría, no sería claro si existiría un incremento o disminución de las horas trabajadas. Sin embargo, dada la baja flexibilidad en el margen intensivo del mercado laboral chileno ${ }^{9}$ sobre la factibilidad de poder reducir horas de trabajo (Rau 2012), es de esperar que en general no haya ajustes a la baja en el número de horas trabajadas por parte de quienes ya estuviesen trabajando.

Debemos señalar también que existe evidencia bastante robusta acerca de la efectividad del EITC en otras partes del mundo. Por ejemplo, Eissa y Liebman (1996) muestran, mediante un análisis de diferencias en diferencias, aumentos de la tasa de participación de mujeres solteras en Estados Unidos luego de la implementación de un impuesto negativo al ingreso. Este resultado es confirmado por Meyer y Rosenbaum (2001).

\footnotetext{
${ }^{9}$ Sumada a la baja penetración del trabajo a tiempo parcial.
} 

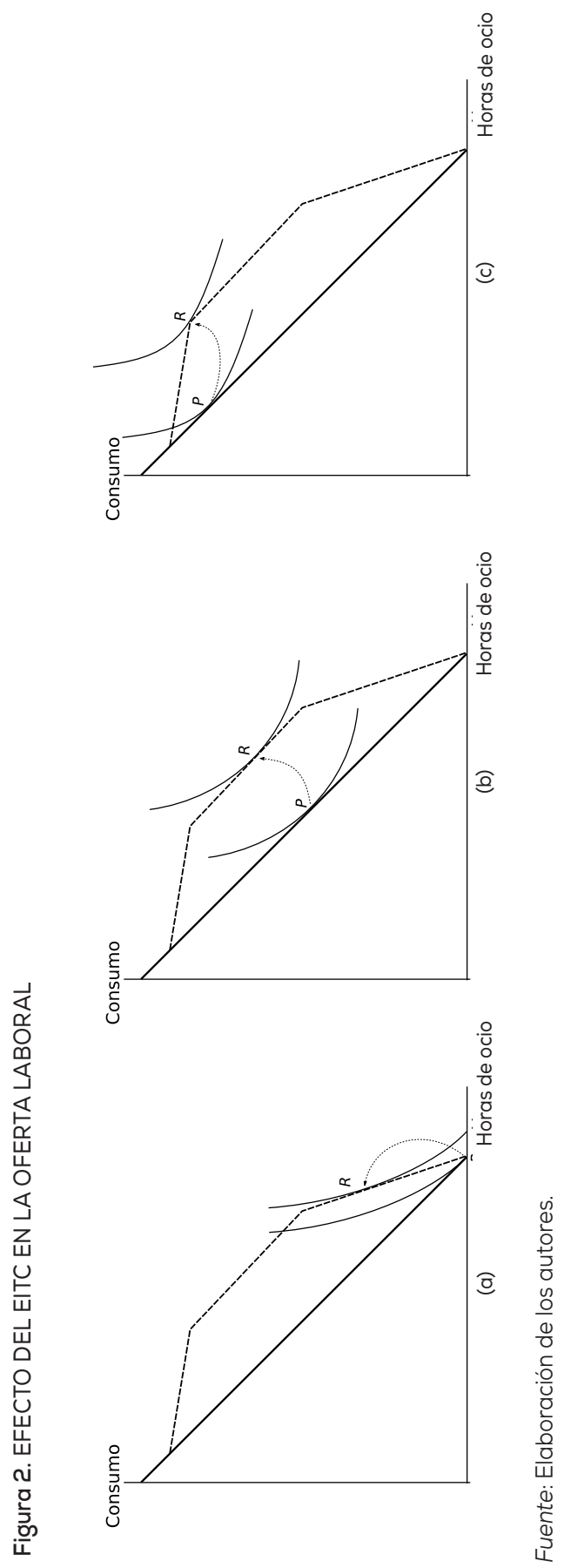


\section{MÉTODO DE SIMULACIÓN Y OPTIMIZACIÓN10}

Nuestro propósito es encontrar, para cada presupuesto posible, el diseño del INIT que maximiza la disminución en el índice de Gini. Esto requiere de dos procesos distintos anidados: uno que simule, para cada combinación considerada de los parámetros del INIT (y las restricciones que entre éstos puedan definirse), el efecto en el índice de desigualdad de ingresos. Luego, un segundo algoritmo que optimice eligiendo aquellos parámetros que permiten conseguir el mejor resultado posible.

Nuestro método de simulación es muy similar al planteado por Agostini et al. (2013): este procedimiento requiere de una serie de pasos que construyen secuencialmente la simulación final del efecto del pago del beneficio.

Primero, la definición del beneficio debe considerar qué ingresos son los que se considerarán para la calificación y pago del subsidio. Por tratarse de un subsidio a los ingresos laborales, el pago del mismo debe depender de los ingresos derivados del trabajo de los individuos; así, consideramos como base para el cálculo del subsidio al mayor entre los ingresos imponibles y los ingresos laborales.

Segundo, un subsidio de este tipo incrementa los retornos esperados del trabajo, lo cual motiva a algunas personas que no están trabajando a incorporarse al mercado laboral. Para poder simular esto, necesitamos estimar dos datos que no observamos: la probabilidad de que alguien que no está trabajando decida comenzar a trabajar y el salario (y, por consiguiente, el subsidio) al que podría aspirar por el hecho de hacerlo.

En una tercera etapa, es necesario determinar quiénes calificarían para el cobro del subsidio y en qué monto lo harían de acuerdo al dise-

${ }^{10}$ Nuestras simulaciones usan los datos de la encuesta Casen 2013 y los ingresos de la metodología tradicional. A partir de 2013 se eliminó el ajuste por cuentas nacionales de los ingresos, por lo que éstos deberían estar subestimados por la encuesta. En consecuencia, es posible que nuestros cálculos resulten en una sobreestimación de los costos del programa. El efecto en desigualdad, por otra parte, es menos claro. Bravo y Valderrama (2011) muestran que el ajuste por cuentas nacionales tiene el efecto de elevar el índice de Gini, ya que gran parte del ajuste amplifica las corrientes de ingresos más importantes para las familias más ricas. Sin embargo, esto no es suficiente para pensar que la variación inducida por el INIT pudiera estar sobreestimada o subestimada al no considerar el ajuste. 
ño de la política propuesta, las características del hogar y los ingresos imputables y laborales calculados en la primera etapa o potenciales (computados en el segundo paso), en el caso de quienes no se encuentran trabajando.

En cuarto término, es necesario determinar cuáles de los individuos que estando fuera de la fuerza de trabajo formal se incorporarían a la vida laboral. Para esto se usan las probabilidades calculadas en la segunda etapa y la variación en las mismas inducida por la creación del subsidio (supuesto como el único cambio relevante en las condiciones laborales de los individuos). Con estos cambios en las probabilidades se determina el cambio en la población activa y se usan las probabilidades finales de trabajar para decidir quiénes son los individuos que buscarán trabajo.

Finalmente, simulados los ingresos y los pagos del subsidio de cada individuo, es necesario agregar estos pagos a nivel de hogar y usar esta información para computar los cambios en los indicadores sociales relevantes (por ejemplo, pobreza extrema, pobreza, desigualdad de ingresos) que se pretenden afectar con la política.

Para el proceso de optimización, dado que el efecto en el Gini no es propiamente diferenciable para cada parámetro, optamos por un mecanismo de búsqueda aleatorio en la forma de un algoritmo genético que no requiere el cálculo de derivadas ni analíticas ni numéricas y utiliza sólo una evaluación de la política en cada ciclo.

Lo que queda de esta sección describe, en mayor detalle, cómo se implementó cada una de estas etapas y las decisiones que se tomaron al respecto.

\subsection{Construcción del ingreso imponible}

Los ingresos imponibles de los individuos se construyeron a partir de los ingresos autónomos registrados en la encuesta Casen, ${ }^{11}$ descontando aquellas partidas que no constituyen ingresos laborales o que se descuentan de estos para cálculos de la base imponible.

${ }^{11}$ Los ingresos autónomos son una variable construida por el Ministerio de Desarrollo Social a partir de las diversas corrientes de ingresos consideradas en la encuesta. Nuestra "construcción", en rigor, sólo descuenta de esta variable algunas corrientes que no son parte de la base tributaria. 
En particular, de la variable de ingresos autónomos descontamos el autoconsumo de productos agrícolas, por cuanto no constituye un ingreso laboral monetario tributable, y el retiro de productos desde el negocio propio, en el caso de independientes, por la misma razón anterior. Se descuentan, además, las siguientes partidas que no constituyen ingresos laborales: el arriendo de propiedades urbanas, agrícolas y por temporadas, los intereses por depósitos y el cobro de dividendos por inversiones (acciones o bonos financieros).

En el caso de individuos que trabajan a honorarios, descontamos también el gasto presunto (30 por ciento de los ingresos por honorarios), con un tope de 15 unidades tributarias anuales (UTA).

El ingreso remanente así construido constituye nuestra estimación del ingreso imponible individual sobre el que se calcula, cuando corresponde, el impuesto negativo (INIT).

\subsection{Cálculo de la probabilidad de trabajar y del salario esperado}

Para todos las personas en edad de trabajar ${ }^{12}$ se estiman, mediante el método de Heckman (1979) en dos etapas, la probabilidad de estar trabajando y el (logaritmo del) salario por hora esperado por trabajar. La estimación excluye a los núcleos familiares de las trabajadoras y trabajadores de servicio doméstico puertas adentro, ${ }^{13}$ así como a todos los adultos de los núcleos en los que existen personas que trabajan pero no reportan ingresos del trabajo o que trabajan pero no reportan horas trabajadas semanalmente en el mes de referencia (ya sea directamente o de acuerdo a las horas estipuladas en su contrato o acuerdo de trabajo). ${ }^{14}$ Los resultados de estas estimaciones se encuentran en el apéndice 1.

La variable dependiente considerada es el logaritmo natural del salario por hora (salario mensual dividido por horas semanales de trabajo reportadas multiplicadas por 4,2). Las variables independientes

${ }^{12}$ Hombres entre 18 y 65 años, y mujeres entre 18 y 60 años.

${ }^{13}$ Núcleo 0 en la nomenclatura de Casen.

${ }^{14}$ La exclusión de los núcleos completos y no sólo de las observaciones con problemas intenta reducir el uso de observaciones potencialmente problemáticas, por cuanto un adulto cuya información laboral esté incompleta o sea inconsistente puede indicar problemas en la aplicación de la encuesta completa (o, al menos, de los módulos laborales y de ingresos que son los más usados en esta parte del análisis). 
incluyen: la educación del individuo medida en años de escolaridad; la experiencia laboral potencial del individuo (diferencia entre la edad y la educación formal y los primeros seis años de vida); el cuadrado de la experiencia potencial; los ingresos de otros individuos del hogar y variables categóricas para los individuos que residen en la zona urbana, y también los que residen en la Región Metropolitana; el nivel educacional de la madre (primaria, media o superior); el nivel educacional del padre (primaria, media o superior); y el sexo del individuo. Los datos del nivel educacional de la madre y del padre provienen del módulo autobiográfico de la encuesta.

Para corregir por el sesgo de selección inherente - que consiste solamente en observar salarios para los individuos que efectivamente trabajan-, el modelo de Heckman estima en paralelo un modelo de elección discreta de participación laboral. Las variables que incluimos en esta ecuación de selección (cuya variable dependiente vale 1 si el individuo trabaja y 0 si no lo hace) incluyen la escolaridad, la edad del individuo, el cuadrado de su edad, los ingresos de otros miembros del hogar y un conjunto de variables categóricas para la zona urbana o rural, la región de residencia (Metropolitana u otra), el nivel educacional del padre y de la madre, el sexo del individuo, su condición de discapacidad (variable que vale 1 si el individuo sufre de una condición permanente que reduce su movilidad), su estado civil (1 si está separado o divorciado y otra variable si es viudo) y variables binarias para la presencia en el hogar de: (i) otros ocupados; (ii) adultos jóvenes que ni estudian ni trabajan; (iii) discapacitados; y niños dependientes: (iv) entre 0 y 2 años; (v) entre 3 y 5 años; (vi) o entre 6 y 12 años de edad respectivamente.

De esta estimación se producen tres resultados: un estimador consistente del salario por hora potencial para los individuos que no trabajan; un estimador consistente de la probabilidad $\phi i$ de que cada individuo $i$ que no trabaja lo haga; y un estimador consistente del efecto marginal $\phi_{i}$ de que el mismo individuo trabaje.

\subsection{Determinación de los individuos que calificarían para el subsidio y el monto que cobrarian}

De acuerdo al diseño de nuestro subsidio, clasifican al INIT todos los individuos con un ingreso imponible (o predicho, en caso de no tra- 
Tabla 2. PARÁMETROS EMPLEADOS PARA LA CONSTRUCCIÓN DEL INIT

\begin{tabular}{ll}
\hline Parámetro & Descripción \\
\hline Intercepto $\mathrm{O}\left(\mathrm{I}_{\mathrm{O}}\right)$ & $\begin{array}{l}\text { Monto fijo recibido (independiente de los } \\
\text { ingresos del trabajo), para trabajador sin } \\
\text { cargas. }\end{array}$ \\
Intercepto $\mathrm{H}\left(\mathrm{I}_{\mathrm{h}}\right)$ & $\begin{array}{l}\text { Monto inicial recibido (independiente de } \\
\text { los ingresos del trabajo) por cada hijo } \\
\text { (hasta } 3) .\end{array}$ \\
Inicio sección plana $\left(\mathrm{I}_{\mathrm{sp}}\right)$ & $\begin{array}{l}\text { Ingreso para el que la transferencia deja } \\
\text { de crecer. }\end{array}$ \\
Ancho sección plana $\left(\mathrm{A}_{\mathrm{sp}}\right)$ & $\begin{array}{l}\text { Intervalo de ingresos por el que la trans- } \\
\text { ferencia se mantiene constante. } \\
\text { Techos }\left(\mathrm{T}_{\mathrm{i}}\right)\end{array}$ \\
Tasas de retiro $\left(\mathrm{R}_{\mathrm{i}}\right)$ & $\begin{array}{l}\text { Monto máximo que alcanza la transferen- } \\
\text { cia, dependiendo del número de cargas. } \\
\text { Tasa a la que decrece la transferencia } \\
\text { para ingresos mayores al inicio de la sec- } \\
\text { ción plana más su ancho. }\end{array}$ \\
\hline
\end{tabular}

bajar) en el tramo exento del impuesto a la renta. La cobertura ${ }^{15}$ se controla endógenamente, de modo de respetar la restricción presupuestaria. Además se toma en cuenta el número de cargas presentes en el hogar, considerándose como tales a menores de 18 años, a jóvenes entre 18 y 24 años que se encuentren estudiando y a miembros del hogar que presenten alguna discapacidad importante. ${ }^{16}$

A continuación, se procede a calcular el monto de INIT que podrían cobrar todos quienes califiquen para el beneficio. Los parámetros que definen los montos y tramos del subsidio (particularmente tasas de crecimiento y retiro, monto de corte del beneficio; así como también inicio y ancho de la sección plana, entre otros) son las variables sobre las que se efectúa la búsqueda del diseño óptimo, para cada presupuesto posible. La tabla 2 muestra un listado completo de los parámetros posibles, sobre los cuales opera el algoritmo de optimización. Más adelante se especifican las restricciones que se impusieron a éstos. La figura 3 muestra cómo estos parámetros permiten el cálculo del pago para cualquier nivel de

${ }^{15}$ En términos del porcentaje de los hogares de menores ingresos que pueden participar del programa.

${ }^{16}$ En el caso de un hogar que tenga más de un adulto trabajando o que potencialmente podría trabajar, se asignaron el total de cargas al jefe de hogar, en forma indivisible. Otro adulto que trabaje o pueda trabajar todavía puede ser beneficiario del INIT, pero no puede cobrar por las cargas del hogar. 
Figura 3. CÁLCULO DEL INIT A PARTIR DE SUS PARÁMETROS

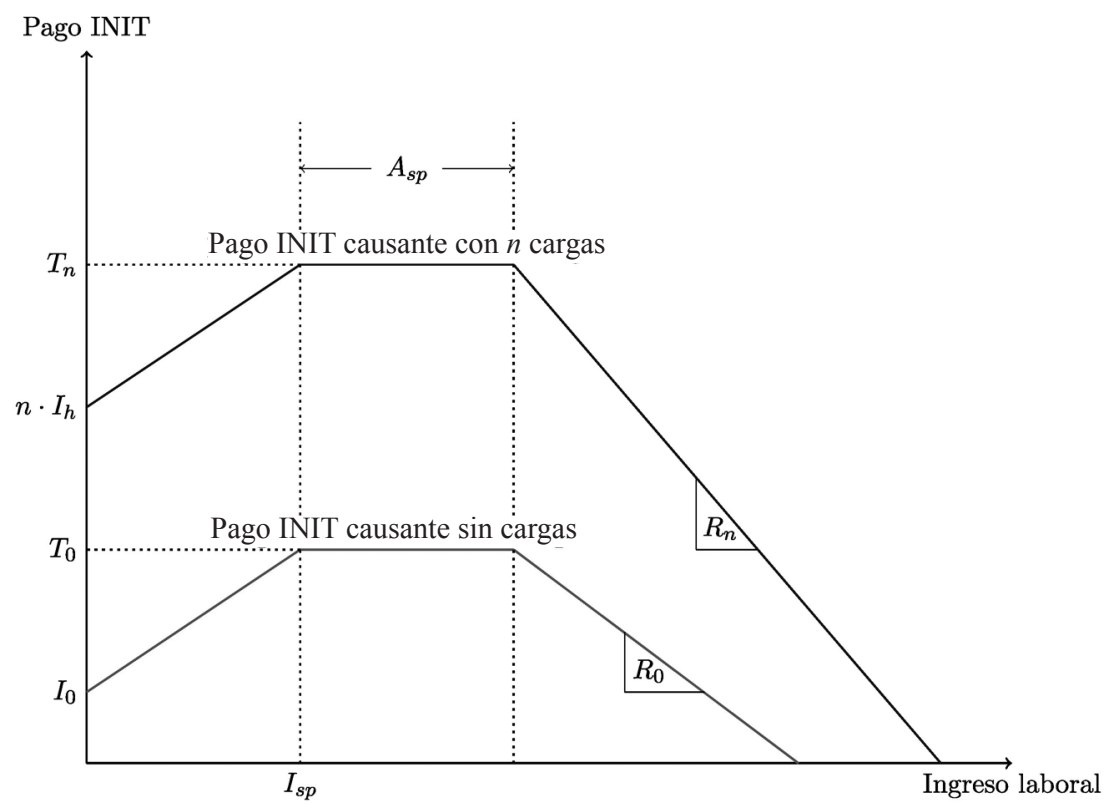

Fuente: Elaboración de los autores.

ingresos. En algunos casos optamos por codificar, en vez del techo (o sea, el monto máximo de transferencia), la tasa de crecimiento de la transferencia $\left(\mathrm{C}_{\mathrm{i}}\right)$, es decir, la pendiente del tramo creciente. ${ }^{17}$

Para calcular el monto del subsidio para aquellas personas que califican pero que no trabajaban con anterioridad a la instauración del programa, se realizaron imputaciones de los salarios por hora predichos mediante el método de Heckman, asumiendo que ingresaban al mercado laboral trabajando 45 horas semanales. A diferencia de los actualmente existentes subsidios al empleo, que distribuyen el pago del beneficio entre trabajador y empleador, en este caso consideramos que el total del aporte va dirigido al trabajador, lo que es consistente con el diseño y espíritu de las transferencias que complementan a la autogeneración de ingresos.

${ }^{17}$ Ambas especificaciones son equivalentes, dado que, para cualquier combinación $\mathrm{I}_{\mathrm{i}}, \mathrm{T}_{\mathrm{i}}, \mathrm{I}_{\text {sp }}$ se cumple que $\mathrm{C}_{\mathrm{i}}=\left(\mathrm{T}_{\mathrm{i}}-\mathrm{I}_{\mathrm{i}}\right) / \mathrm{I}_{\mathrm{sp}}$ 


\subsection{Determinación de nuevas incorporaciones al mercado laboral}

El paso siguiente corresponde a la asignación efectiva del beneficio. Para esto es necesario identificar a los potenciales beneficiarios que podrían incorporarse al mercado del trabajo como respuesta al establecimiento del INIT. Para simular esta incorporación primero se ordenan los individuos que originalmente no trabajaban de acuerdo a la probabilidad de participación ex post predicha, la que en su cálculo requiere de una estimación de la elasticidad de la oferta de trabajo.

No existen buenos estimadores de la elasticidad del trabajo en Chile, debido a lo cual usamos un valor de la elasticidad estimada en la literatura para la economía de Estados Unidos y que es de 0,3. ${ }^{18}$ Esto quiere decir que, por cada 10 por ciento que aumenta el salario real promedio, la oferta de trabajo se expande en un 3 por ciento. Existe, sin embargo, una importante distinción entre la elasticidad "macro" del trabajo, que tiene la interpretación aquí presentada, y la elasticidad "micro", que representa el cambio en probabilidad de que una persona que no está trabajando decida hacerlo a partir de un cambio en el ingreso. ${ }^{19}$ Así, por ejemplo, si muchas personas se encuentran (debido, v. g., a indivisibilidades en la jornada laboral) justo en el margen respecto a trabajar y no hacerlo, un cambio en el ingreso puede inducir una pequeña variación en la probabilidad individual de trabajo y tener un efecto importante en la cantidad de personas que deciden trabajar. Nuestro supuesto es sobre la elasticidad "macro", lo que requiere de algún cálculo extra para derivar la elasticidad "micro" correspondiente (Heckman 1993). En el apéndice 2 se muestra cómo obtenemos un estimador de la elasticidad "micro" $\gamma_{k}$ a partir de la elasticidad "macro" $\varepsilon_{k}{ }^{20}$ Con este

${ }^{18}$ Véase Eissa y Hoynes (2011) o Chetty et al. (2011).

${ }^{19}$ Consideramos aquí que el cambio fundamental en la oferta de trabajo ocurre en el margen extensivo, es decir, en la cantidad de gente que trabaja y no en el número de horas que se trabaja. Este supuesto se sustenta en la evidencia citada anteriormente (Rau 2012).

${ }^{20}$ En este punto nuestro análisis difiere del de Agostini, Selman y Perticará (2013), quienes aproximan la probabilidad de incorporación por:

$$
\operatorname{Prob}\left(S_{I N I T}=1\right)=\Phi\left(Z_{i} \gamma\right)+\phi\left(Z_{i} \gamma\right) \cdot \varepsilon_{k} \cdot \log \left(\Delta y_{i}\right)
$$

dónde $Z_{i}$ son las características que determinan la participación laboral del individuo, $\varepsilon_{k}$ es la elasticidad "macro" de la oferta de trabajo y $\Delta \gamma_{i}$ es el cambio en el ingreso laboral por incorporación (suma de la remuneración esperada y aporte del INIT menos los beneficios de desempleo que podría estar cobrando). 
estimador de la elasticidad individual, el cambio predicho en la probabilidad personal de trabajar, de aquellos que no lo hacen, está dado por:

$$
\Delta \operatorname{Prob}\left(S_{I N I T}=1\right)=\phi\left(Z_{i} \gamma\right) \cdot \gamma_{k} \Delta \ln \left(Y_{i}\right)
$$

Donde $\phi\left(Z_{i} \gamma\right)$ corresponde al valor marginal estimado en la segunda etapa, y $\Delta \ln \left(Y_{i}\right)$ es el cambio en el logaritmo natural del ingreso esperado del individuo que puede impetrar el cobro de INIT, esto es:

$$
\Delta \ln \left(Y_{i}\right)=\ln \left(W_{i}+I N I T_{i}-D_{i}\right)-\ln \left(W_{i}-D_{i}\right)
$$

En esta expresión, $W_{i}$ es el salario predicho del individuo $i, I N I T_{i}$ el cobro al que tendría derecho por el programa y $D_{i}$ los beneficios de cesantía que esté cobrando.

Finalmente, se considera que se incorporan al mercado laboral (y por lo tanto se beneficiarán del INIT) las personas con mayor probabilidad estimada de trabajar $\Phi_{i}$, con un corte que se calcula usando la variación predicha en la participación laboral agregada, es decir:

$$
\Delta L=\sum_{i} \Delta \operatorname{Prob}\left(S_{I N I T}=1\right)=\sum_{i} \phi\left(Z_{i} \gamma\right) \cdot \gamma_{k} \Delta \ln \left(Y_{i}\right)
$$

\subsection{Cálculo del efecto en desigualdad}

Considerando entonces como beneficiarios del INIT a todos los que clasifican para el mismo y que ya trabajaban $-\mathrm{y}$ a los que se integrarán al mercado laboral como resultado de la creación del beneficio_-, se calcula la nueva distribución de ingresos. Para esto, al ingreso monetario del hogar se suma el monto del INIT que recibiría dicho hogar descontados los beneficios de cesantía que se dejarían de percibir producto de haber ingresado al mercado laboral, según correspondiese. Luego, se calcula el nuevo ingreso monetario per cápita sobre el cual se observan las medidas de desigualdad.

Por último, es importante señalar que el análisis que presentamos asume que no hay ajuste de horas laborales de las personas que ya trabajaban antes del programa.

\subsection{Optimización}

El objetivo de este ejercicio es encontrar el diseño de impuesto negativo al ingreso del trabajo que produce la máxima reducción en 
desigualdad para un presupuesto determinado. Dado que la función objetivo resulta de una simulación sobre los efectos de la política en toda la población y no tiene, por lo tanto, una expresión analítica, no es posible obtener el óptimo directamente y debemos recurrir a métodos numéricos de optimización.

Por otra parte, la evaluación de la función objetivo es particularmente lenta en nuestro caso, por lo que optar por métodos numéricos que determinan direcciones de crecimiento utilizando diferenciación numérica tampoco parecía una buena estrategia. Esto nos obliga a emplear algoritmos de optimización que no usan diferencias.

Dentro de este tipo de algoritmos, optamos por usar un algoritmo genético, esto, principalmente, porque los algoritmos genéticos se prestan directamente para modelar situaciones en que algunas de las variables sobre las que se optimizan son discretas y, además, reducen significativamente el número de evaluaciones de la función objetivo requeridas.

Un algoritmo genético es un método de búsqueda global estocástica, que obtiene su nombre del hecho de simular parcialmente la metáfora del proceso evolutivo biológico. Al inicio del proceso se forma una población de individuos al azar, cada uno de los cuales representa una potencial solución al problema que está codificado en una serie de cromosomas que representan los valores de las variables (en este caso, los parámetros del INIT). Cada una de estas potenciales soluciones es evaluada en la función objetivo para obtener la adaptación del individuo a su ambiente (acá la presión evolutiva es definida por la función objetivo). Luego, utilizando iterativamente el principio de sobrevivencia del más apto, el mecanismo aspira a generar aproximaciones de calidad incremental a la solución del problema de optimización planteado (ver Chipperfield et al. 1994).

Generada la población original, por un número de generaciones definido, esta población evoluciona de acuerdo a la siguiente estrategia: (1) un número de individuos mejor adaptados es preservado a la generación siguiente; (2) se forman nuevos individuos para completar la población. Así, se eligen dos individuos al azar con una probabilidad que depende de su evaluación en la función objetivo (los con mejores resultados tienen una probabilidad más alta de ser elegidos) y ellos generan un nuevo individuo por recombinación entre sus genes (lo que provoca 
nuevos valores de los parámetros a ser evaluados); (3) se introducen mutaciones a la población en la forma de alteraciones a algunos genes de algunos individuos, alteraciones que son provocadas aleatoriamente. El objetivo de la mutación es introducir variabilidad artificial en la población y asegurarse de que no existan regiones del espacio factible que no sean exploradas con alguna probabilidad; ${ }^{21}$ y (4) se evalúan los nuevos individuos en la función objetivo y se procede a la siguiente generación.

Cuando la población inicial es generada adecuadamente, este algoritmo identifica primero algunos óptimos locales y luego, en forma azarosa, va repoblando y explorando las áreas más prometedoras del espacio estudiado, encontrando, en cada iteración, la mejor respuesta de la población completa.

Por supuesto, es necesario efectuar algunas elecciones respecto a la implementación del algoritmo y es necesario también explorar si las respuestas son sensibles o no a estas elecciones. En nuestro caso, en los resultados presentados, usamos un tamaño de la población de 80 individuos. Nuestra opción fue generar por reproducción sexual al 80 por ciento de ellos en cada generación, permitimos que hasta el 70 por ciento del genoma de un "hijo" fuera transmitido desde un único "padre" y dejamos a la población evolucionar por 250 generaciones. $^{22}$

La solución fue implementada en el software Octave usando el Genetic Algorithm Toolbox de la Universidad de Sheffield, programa implementado por Chipperfield et al. (1994).

El problema que nos interesa incluye algunas restricciones (más notablemente, la restricción presupuestaria, pero también otras asociadas a, por ejemplo, evitar que los pagos entre individuos en grupos distintos se intersecten entre sí), las que deben ser integradas al problema. En la mayoría de los casos, si las restricciones pueden incorporarse, ya sea a la codificación del espacio factible o en la función objetivo, es de-

${ }^{21}$ Siendo el proceso de mutación aleatorio, esto sólo garantiza que haya una probabilidad de explorar todo el espacio factible sin importar la forma de la población inicial. Por supuesto, esto no asegura que, para una población inicial específica, ésta pueda evolucionar sin explorar algunas regiones.

22 En optimizaciones no reportadas variamos el tamaño de la población entre 50 y 125 individuos y el número de generaciones, entre 100 y 500 . Los resultados no cambiaron en modo sensible. Todos los programas utilizados están disponibles solicitándolos a los autores. 
seable que se haga (Reid 1996). De lo contrario, es necesario alterar el mecanismo de evaluación para mantener al grupo relevante de la población (donde se encuentra el óptimo restringido) dentro del espacio factible (ver, por ejemplo, Petridis et al. 1998; Wah y Chen 2000; Yeniay y Ankara 2005).

En nuestro caso, las restricciones son relativamente fáciles de tratar. En primer lugar, la restricción presupuestaria se integra a la función objetivo endogeneizando la cobertura. Así, para cada valor de los parámetros del INIT y presupuesto posible, por construcción se decide calificar a las personas según el ingreso autónomo de sus hogares en forma creciente, de ese modo, siempre se sirve al porcentaje de la población de menores ingresos, que permiten los recursos disponibles, ${ }^{23}$ esto garantiza que, en cada solución, se satisface la restricción presupuestaria. Una segunda restricción (que no resulta activa nunca) es que los beneficios no se extiendan más allá del séptimo decil, es decir, que no se beneficien individuos en el 30 por ciento de más altos ingresos de la población.

Segundo, cuando decidimos restringir el análisis a tasas de crecimiento y retiro que implican que un causante con más cargas siempre reciba una transferencia mayor que otro causante con menos cargas, esto se modela directamente en la representación de los parámetros en los individuos, de modo que las trayectorias de beneficios tengan las mismas tasas para todos los perfiles de causantes posibles.

En la especificación que mostramos a continuación usamos las siguientes restricciones en el proceso de optimización: primero, fijamos el intercepto para un beneficiario sin cargas a 0 , de modo que un beneficiario que genera escasos ingresos no experimenta un salto inicial en su cobro. El monto a cobrar por cada carga (con un máximo de 3) lo restringimos entre $\$ 30.000$ y $\$ 75.000$ por carga. Restringimos el inicio de la sección plana a un monto de ingresos entre $\$ 50.000$ y $\$ 150.000$, la tasa de crecimiento del cobro (para ingresos entre $0 \mathrm{y}$ el inicio de la sección plana) la restringimos entre 0,25 y 1,00 , y, finalmente, restringimos la tasa de retiro a cualquier valor entre $-0,75$ y $-0,25$.

El objetivo de estas restricciones es preservar la forma del beneficio que hemos presentado en la figura 3 y asegurar que dos o más

${ }^{23}$ Además de ser una implementación computacionalmente conveniente, ésta es la forma en que tradicionalmente se focalizan las políticas públicas en Chile. 
curvas de beneficios para causantes con distinto número de cargas no se crucen. Esto permite una política que es más realista, cuya implementación sea políticamente factible y fácilmente comunicable. ${ }^{24}$

\section{RESULTADOS DE LA SIMULACIÓN}

Presentamos, a continuación, los resultados de las distintas optimizaciones realizadas en diversos ejercicios. Se muestran, en primer lugar, una curva de caída de la desigualdad para distintos niveles de gasto en el INIT, asumiendo que no existe adición de nuevos trabajadores al mercado laboral. Éstos son los resultados directos de nuestro algoritmo de optimización. Luego, presentamos otras dos curvas como la anterior, pero que asumen dos valores posibles de la elasticidad laboral. Estos resultados se obtienen simulando nuevamente a partir de las salidas de la primera curva y es la razón por la que presentamos la curva sin aditividad en primer lugar.

Otra razón por la que la primera curva es interesante está en que representa una cota inferior de la disminución de la desigualdad para el diseño del INIT implícito (no para el presupuesto considerado, pues éste puede aumentar al considerar incorporación de nuevos trabajadores).

\subsection{Caso 1: Sin aditividad de trabajadores al mercado laboral}

La tabla 3 presenta el número de personas que, de acuerdo al criterio de calificación descrito (personas que tienen ingresos imponibles en el tramo exento y pertenecen a uno de los primeros siete deciles de ingresos autónomos) podrían tener derecho a cobrar el INIT, sin considerar el potencial efecto de un aumento de la participación laboral (o, lo que es lo mismo, asumiendo que o que ninguno de los trabajadores que se incorpora encuentra un trabajo). Los resultados se muestran desagregados según el número de cargas por el que podrían cobrar.

Como se observa, el número de beneficiarios que podrían cobrar el INIT ascendería a 3.575.478, de los cuales cerca de 1,5 millones cobra-

${ }^{24}$ En ejercicios no reportados, disponibles si se solicitan a los autores, realizamos simulaciones en las que levantamos algunas de estas restricciones. El efecto en el índice de desigualdad no es muy relevante y las formas de los diseños que se obtienen son difíciles de defender en términos de política pública. 
Tabla 3. POTENCIALES COBRADORES DE INIT, SEGÚN NÚMERO DE CARGAS

\begin{tabular}{lrrr}
\hline & Califican & Porcentaje & Acumulado \\
\hline Sin cargas & 2.078 .351 & 58,13 & 58,13 \\
Una carga & 771.731 & 21,58 & 79,71 \\
Dos cargas & 517.913 & 14,49 & 94,20 \\
Tres o más cargas & 207.483 & 5,80 & 100,00 \\
Total & 3.575 .478 & 100,00 & \\
\hline
\end{tabular}

Fuente: Cálculos de los autores empleando encuesta CASEN 2013.

Tabla 4. PARÁMETRO INIT ÓPTIMO PARA DISTINTOS NIVELES DE PRESUPUESTO

\begin{tabular}{|c|c|c|c|c|c|c|c|c|}
\hline $\begin{array}{l}\text { Presu- } \\
\text { puesto } \\
\text { [MMUS\$] }\end{array}$ & $\begin{array}{c}\text { Cober- } \\
\text { tura } \\
{[\%]}\end{array}$ & $\begin{array}{l}\mathrm{I}_{0} \\
{[\$]}\end{array}$ & $\begin{array}{l}I_{h} \\
{[\$]}\end{array}$ & $\begin{array}{l}\mathrm{I}_{\mathrm{sp}} \\
{[\$]}\end{array}$ & $\begin{array}{l}A_{s p} \\
{[\$]}\end{array}$ & $C_{i}$ & $\mathrm{R}_{\mathrm{i}}$ & $\begin{array}{c}\text { Gini } \\
\text { [L.B. }=0,5165]\end{array}$ \\
\hline 100 & 5 & 0 & 30.209 & 44.877 & 19.714 & 0,98 & $-0,28$ & 0,5149 \\
\hline 200 & 7 & 0 & 30.016 & 62.488 & 27.835 & 0,98 & $-0,33$ & 0,5133 \\
\hline 300 & 9 & 0 & 30.055 & 62.382 & 27.780 & 0,98 & $-0,34$ & 0,5119 \\
\hline 400 & 10 & 0 & 30.042 & 84.826 & 38.752 & 0,87 & $-0,39$ & 0,5104 \\
\hline 500 & 11 & 0 & 30.080 & 94.787 & 43.746 & 0,84 & $-0,42$ & 0,5090 \\
\hline 750 & 13 & 0 & 30.012 & 117.357 & 55.336 & 0,83 & $-0,51$ & 0,5056 \\
\hline 1000 & 16 & 0 & 33.829 & 110.792 & 52.097 & 0,88 & $-0,53$ & 0,5024 \\
\hline 1500 & 19 & 0 & 41.251 & 124.356 & 59.720 & 0,93 & $-0,67$ & 0,4964 \\
\hline 2000 & 23 & 0 & 48.026 & 124.016 & 59.857 & 1,00 & $-0,75$ & 0,4909 \\
\hline 2500 & 28 & 0 & 48.285 & 123.881 & 59.806 & 1,00 & $-0,75$ & 0,4860 \\
\hline 3000 & 33 & 0 & 48.283 & 123.874 & 59.802 & 1,00 & $-0,75$ & 0,4816 \\
\hline 3500 & 39 & 0 & 48.018 & 124.251 & 59.983 & 1,00 & $-0,75$ & 0,4778 \\
\hline 4000 & 44 & 0 & 47.994 & 124.284 & 60.000 & 1,00 & $-0,75$ & 0,4746 \\
\hline 4500 & 50 & 0 & 47.949 & 124.295 & 60.000 & 1,00 & $-0,75$ & 0,4719 \\
\hline 5000 & 56 & 0 & 46.845 & 124.458 & 60.000 & 1,00 & $-0,74$ & 0,4699 \\
\hline
\end{tabular}

Notas: En esta tabla, I corresponde al valor inicial de la transferencia para un beneficiario sin cargas; $I_{h}$ al monto inicial de transferencia por cada carga (con un máximo de 3) para los beneficiarios con cargas; $I_{S P}$, al monto del salario en que se inicia la sección plana de la transferencia (es decir, ésta se hace constante); $A_{S P}$, al ancho de esta sección plana (en términos de los ingresos laborales); $C_{i}$ a la pendiente del tramo creciente de la transferencia (tasa de crecimiento), y $\mathrm{R}_{\mathrm{i}}$ a la pendiente del tramo decreciente (tasa de retiro). (Ver la tabla 2, la figura 3 y la nota 17 para más claridad).

Fuente: Elaboración de los autores. 
rían, además, por las cargas que tengan. Por supuesto, en algunos hogares podría existir más de un beneficiario directo. De hecho, el número de hogares con al menos un cobrador alcanzaría a los 2.381.722. Estos hogares sumarían un poco más de 9.300 .000 potenciales beneficiarios directos e indirectos.

La tabla 4 presenta los resultados de la optimización para nuestro diseño preferido del programa en distintos niveles de presupuesto, así como muestra, para cada nivel de gasto asignado, la cobertura (como porcentaje de la población de menores ingresos autónomos que se beneficia del programa), así como los parámetros especificados en la tabla 4 y el índice de Gini obtenido para los parámetros presentados.

Se aprecia que, a medida que aumenta el presupuesto, la cobertura óptima del INIT va aumentando, pero sin llegar al 70 por ciento de nuestra restricción, por lo que esta restricción no está activa. Para examinar de mejor forma qué ocurre con el diseño de los beneficios al aumentar el presupuesto asignado a la política, recurrimos a un análisis gráfico.

En primer lugar, el gráfico 1 muestra la forma que tiene el cobro mensualizado de INIT según el número de cargas del causante cuando el presupuesto es de 1.000 millones de dólares. Como se aprecia, con las restricciones especificadas, las curvas de pago de acuerdo al ingreso son paralelas entre sí. Por ello, basta con evaluar la evolución de una de estas curvas para tener una buena idea de lo que ocurre con el diseño óptimo a medida que aumenta el presupuesto del programa.

A continuación, el gráfico 2 muestra cómo evoluciona el cobro mensual del diseño óptimo de política para un causante con dos cargas a medida que aumenta el presupuesto asignado al programa.

El análisis en el gráfico 2 muestra que, a medida que aumenta el presupuesto asignado al programa, el diseño del INIT que más reduce la desigualdad $^{25}$ cambia aumentando el monto inicial por carga y desplazando el inicio de la sección plana hacia la derecha; esto eleva el cobro total máximo y obliga a aumentar la tasa de retiro del beneficio (recuérdese que el monto total entregado no puede superar el de rentas exentas de impuesto).

${ }^{25}$ Lo dicho podría ser distinto si es que considerásemos que el INIT pudiera desincentivar de trabajar a algunas personas, teniendo en cuenta que en la sección plana y el tramo de salida el efecto ingreso gana preponderancia. 
Gráfico 1. COBRO MENSUAL DE INIT EN PESOS SEGÚN NÚMERO DE CARGAS DEL CAUSANTE PARA UN PRESUPUESTO DE 1.000 MILLONES DE DÓLARES

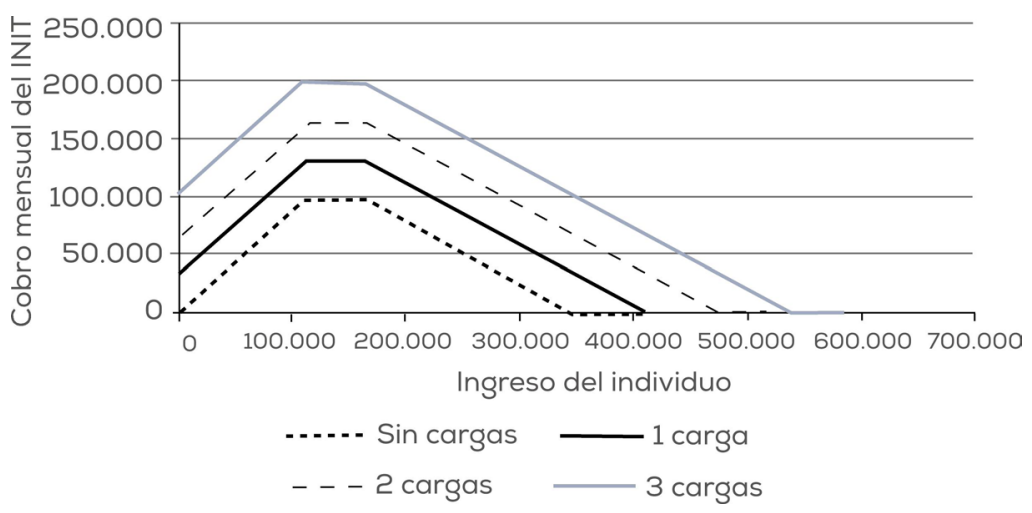

Gráfico 2. COBRO MENSUAL DE INIT ÓPTIMO PARA UN CAUSANTE CON DOS CARGAS PARA DISTINTOS NIVELES DE PRESUPUESTO DEL PROGRAMA

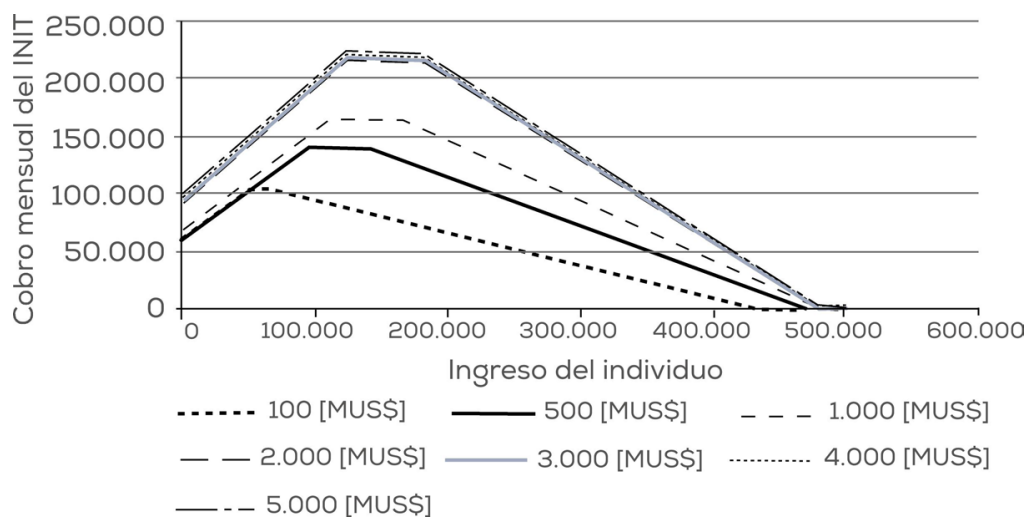

A partir de un presupuesto de 2.000 millones de dólares el programa óptimo se estabiliza en su diseño y sólo aumenta su cobertura. Como muestra el gráfico 3, el aumento de cobertura tiende a crecer a una tasa bastante constante a medida que es mayor el presupuesto asignado. 
Gráfico 3. COBERTURA DEL INIT PARA DISTINTOS NIVELES DE PRESUPUESTO

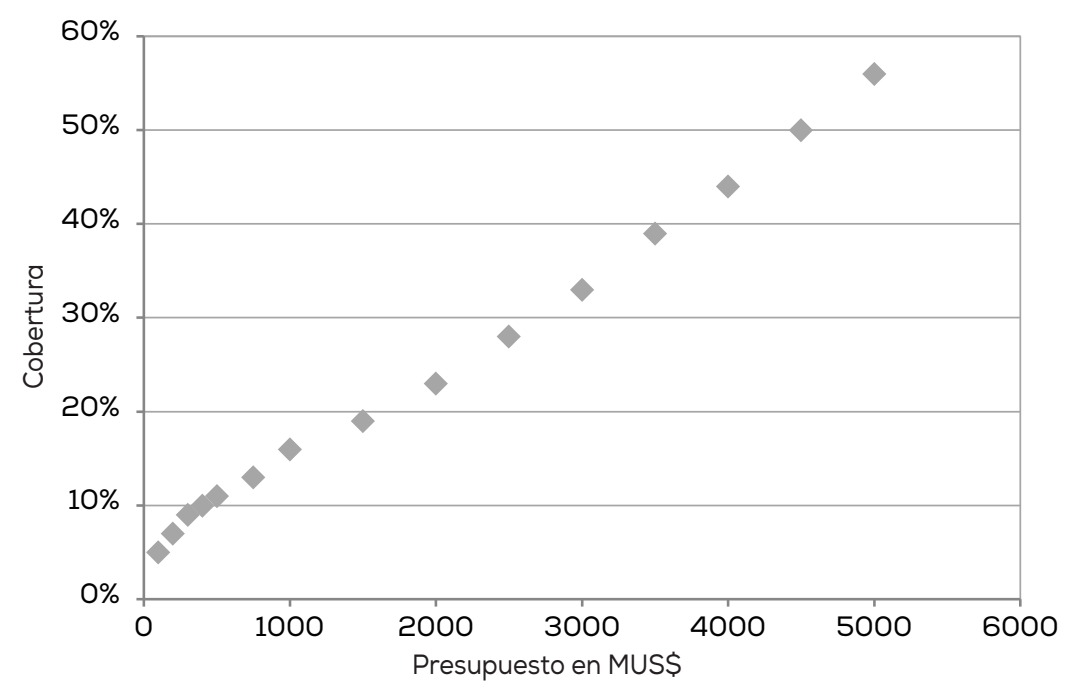

El gráfico 4 muestra la evolución del índice de Gini a medida que se incrementa el presupuesto del programa. Se aprecia que, conforme crece el presupuesto, cae la desigualdad de ingresos total en la economía, aunque lo hace a una tasa decreciente. De hecho, el costo incremental de cada disminución de un punto porcentual en el índice de Gini crece a medida que cae el índice, lo que refleja que cada reducción posterior, en términos relativos, es más costosa que las anteriores.

El coeficiente de Gini cambia desde un valor inicial ${ }^{26}$ de 0,5165 a un mínimo de 0,4699, es decir, una reducción de 4,7 puntos para un gasto de 5.000 millones de dólares, lo que significa un costo promedio de 1.072 millones de dólares por cada punto de disminución de la desigualdad. Sin embargo, como ya se dijo, la caída inicial en la misma es más alta. Los primeros 1.000 millones de dólares invertidos en la política de INIT reducen el Gini en 1,4 puntos.

Un resultado que emerge de este análisis es que los puntos de gasto/Gini que se obtienen de los sucesivos ejercicios de optimización ajustan muy bien a una curva cuadrática en el gasto. En efecto, si G representa el coeficiente de Gini obtenido y $\mathrm{P}$ el presupuesto asignado

${ }^{26}$ Esta medida del Gini es sobre el ingreso monetario, ya que para medir el efecto del INIT debemos observar los ingresos que incluyen las transferencias del Estado. 
Gráfico 4. COEFICIENTE DE GINI PARA DISTINTOS VALORES DE PRESUPUESTO DEL INIT

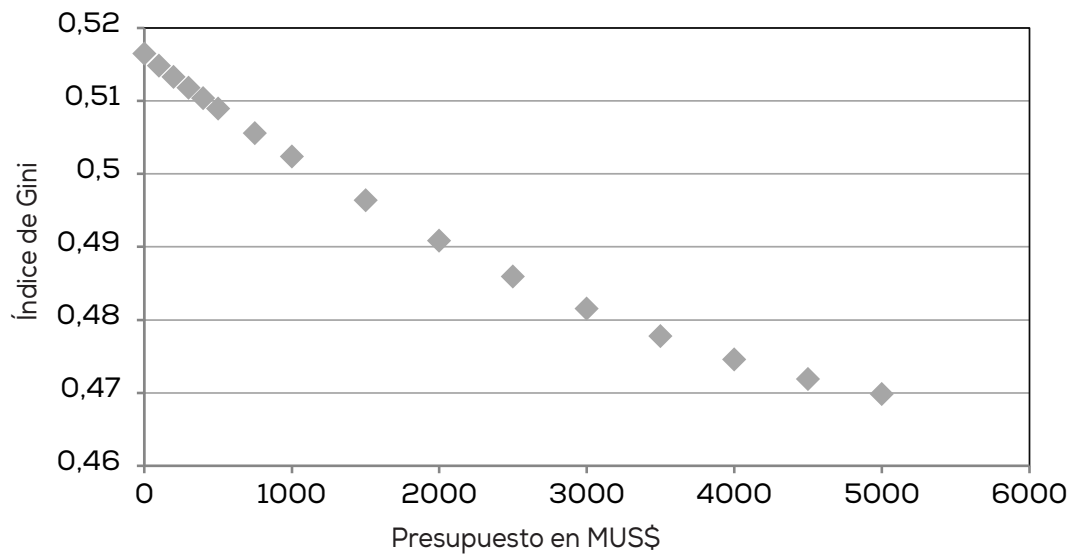

al INIT (en miles de millones de dólares) y estimamos una relación cuadrática entre G y P, obtenemos la siguiente expresión (valores del error estándar entre paréntesis):

$$
G=\underset{(0,0003)}{0,5163}+\begin{array}{r}
0,0011 \\
(0,0000)
\end{array} \quad P^{2}-\underset{(0,0004)}{0,015} P_{R^{2}=0,999}
$$

Una ventaja de esta relación cuadrática es que es bastante simple de entender y extender para puntos intermedios de gasto, lo que facilita el uso de la política de INIT que proponemos como un benchmark o punto de referencia de las políticas de reducción de la desigualdad.

\subsection{Caso 2: Aditividad 'micro' imputando 45 horas de trabajo semanales}

Como ya se dijo, los resultados presentados anteriormente no consideran la posibilidad de que nuevas personas que no están trabajando se incorporen al mundo laboral como respuesta a una política que bonifica los ingresos obtenidos por los trabajadores. Necesariamente incluir este efecto requiere hacer algunos supuestos sobre, primero, el tamaño de esta respuesta (lo que llamamos la elasticidad de oferta del trabajo); luego, sobre la cantidad de personas que conseguirán efectivamente encontrar un empleo y, más aún, sobre el número de horas que decidirán trabajar y los salarios que conseguirán. 
En ciertos casos, algunos de estos supuestos necesarios pueden apoyarse en mediciones anteriores o en los datos disponibles; en otros casos, debemos mantenernos sólo en el terreno de lo especulativo. Con todo, este efecto sólo puede incrementar los resultados presentados, por lo que el lector que no comparta nuestros supuestos puede considerar el resultado anterior como la mejor estimación posible (conservadora en el efecto, mas no en el monto de los recursos necesarios) de los resultados del impuesto negativo al ingreso propuesto en estas líneas.

Para cuantificar el posible efecto de la aditividad al mercado del trabajo, rehacemos nuestras simulaciones, consideramos una elasticidad "micro" de la oferta del trabajo, construida a partir de una elasticidad "macro" de 0,3 tomada de Eissa y Hoynes (2011), como producto de la razón entre la probabilidad de que los que no trabajan lo hagan y el efecto marginal de que los que no trabajan lo hagan. ${ }^{27}$ Asumimos - optimistas - que todas aquellas personas que quisieran trabajar, considerando el efecto del salario y la bonificación asociada al INIT, pueden hacerlo y que los salarios no caen debido a esta incorporación de nuevos trabajadores. En cuanto a las horas que éstos deciden trabajar, asumimos que éstas se mantienen en las 45 horas comunes de la jornada laboral completa.

En las simulaciones que se presentan a continuación (tabla 5) se usan los mismos parámetros del diseño del INIT presentados en la tabla 4, pero agregando el efecto de nuevos trabajadores incorporados a la prestación. El presupuesto base corresponde al monto utilizado en el diseño de la política en la tabla 4. El costo sin adición resulta distinto a este presupuesto, pues adoptamos un diseño de política en que incluimos al total del percentil de corte en la cobertura. ${ }^{28}$

El costo adicional proviene de la incorporación de nuevos trabajadores a la fuerza de trabajo producto del incremento del retorno esperado. Como muestra la tabla, el número de personas que se incorporan a la fuerza de trabajo en nuestras simulaciones crece hasta el presupuesto de 2.000 millones de dólares y luego se estabiliza en torno a 131.000.

${ }^{27}$ Los detalles de este análisis así como la distinción entre elasticidad "macro" y "micro" se explican en la sección anterior.

${ }^{28}$ El diseño óptimo, para respetar la restricción presupuestaria, podía focalizar al interior del percentil. De acuerdo al diseño estándar de políticas públicas en Chile, hemos adoptado la decisión de incluir el total del percentil, lo que eleva los costos aun sin incorporación de nuevos trabajadores. 
Tabla 5. EFECTO Y COSTO DEL PROGRAMA INIT CON INCORPORACIÓN DE NUEVOS TRABAJADORES (EN MILLONES DE DÓLARES).

\begin{tabular}{rrrrrrr}
\hline $\begin{array}{r}\text { Presu- } \\
\text { puesto } \\
\text { (diseño) }\end{array}$ & $\begin{array}{c}\text { Benefi- } \\
\text { ciarios sin } \\
\text { adición }\end{array}$ & $\begin{array}{c}\text { Costo sin } \\
\text { adición }\end{array}$ & Adición & $\begin{array}{c}\text { Costo } \\
\text { adicio- } \\
\text { nal }\end{array}$ & Costo total & Gini \\
\hline 100 & 103.263 & 117,51 & 36.148 & 28,24 & 145,75 & 0,5127 \\
200 & 170.387 & 233,66 & 58.149 & 56,72 & 290,38 & 0,5099 \\
300 & 239.706 & 317,39 & 58.282 & 57,00 & 374,39 & 0,5086 \\
400 & 280.215 & 427,94 & 77.881 & 95,67 & 523,61 & 0,5059 \\
500 & 319.643 & 519,81 & 87.395 & 122,27 & 642,08 & 0,5041 \\
750 & 404.427 & 770,11 & 108.960 & 193,92 & 964,03 & 0,4991 \\
1.000 & 537.304 & $1.017,44$ & 108.977 & 194,84 & $1.212,28$ & 0,4959 \\
1.500 & 661.657 & $1.512,33$ & 124.737 & 287,04 & $1.799,37$ & 0,4887 \\
2.000 & 842.247 & $2.045,30$ & 131.643 & 334,10 & $2.379,40$ & 0,4822 \\
2.500 & 1.081 .574 & $2.510,77$ & 131.504 & 334,06 & $2.844,83$ & 0,4776 \\
3.000 & 1.341 .539 & $3.001,59$ & 131.504 & 334,08 & $3.335,67$ & 0,4733 \\
3.500 & 1.656 .275 & $3.572,11$ & 131.846 & 335,68 & $3.907,79$ & 0,4690 \\
4.000 & 1.917 .580 & $4.019,10$ & 131.846 & 335,77 & $4.354,87$ & 0,4661 \\
4.500 & 2.243 .425 & $4.570,54$ & 131.486 & 335,74 & $4.906,28$ & 0,4632 \\
5.000 & 2.549 .503 & $5.041,56$ & 131.776 & 333,46 & $5.375,02$ & 0,4614 \\
\hline
\end{tabular}

Esto ocurre porque, como ya dijimos, luego de este presupuesto, el diseño óptimo de la política sólo aumenta la cobertura y no modifica la estructura de pagos. Luego, los 131.000 trabajadores incorporados provienen, probablemente, de los percentiles inferiores de ingreso, con lo que sería posible que los de percentiles más altos no clasifiquen para el beneficio o el pago del mismo no sea suficientemente alto como para motivarlos a incorporarse a la fuerza de trabajo.

Respecto a la relación cuadrática entre el costo de la política y la desigualdad, verificamos que ésta se sigue cumpliendo al considerar el efecto de la adición. Y es que una regresión del presupuesto del programa (y su cuadrado) en el coeficiente de Gini de estas simulaciones arroja el siguiente resultado:

$$
G=\underset{(0,0002)}{0,5144}+\underset{(0,0000)}{0,0013} \quad P^{2}-\underset{(0,0004)}{0,0166} \quad P R^{2}=0,999
$$




\subsection{Análisis de sensibilidad}

A continuación, presentamos un análisis de sensibilidad en el cual variaremos dos de los supuestos anteriormente utilizados. El primero de ellos dice relación con la cantidad de horas semanales imputadas para los nuevos trabajadores que ingresarían al mercado laboral producto del INIT. El segundo supuesto, en tanto, hace referencia al tratamiento de la elasticidad de la oferta laboral considerada. En la sección anterior presentamos los resultados asumiendo una elasticidad micro y una jornada de 45 horas semanales; a continuación, presentamos los casos restantes (aditividad micro e imputación de 20 horas semanales, aditividad macro e imputación de 45 y 20 horas semanales).

El supuesto de las 20 horas de trabajo semanales obedecería a un régimen de jornada parcial. En tanto que la elasticidad macro consiste en aplicar el supuesto de la elasticidad agregada del trabajo (valor de $\varepsilon_{k}$ ) a la submuestra de los potenciales beneficiarios, sin considerar el efecto individual en la propensión individual a trabajar. ${ }^{29}$

En la tabla 6 se pueden observar los principales resultados del análisis de sensibilidad. De aquí se desprende que, para los casos que consideran un cierto nivel de aditividad de nuevos trabajadores al mercado laboral, la desigualdad, medida en Gini, oscila entre 0,46 y 0,47 (para un presupuesto de poco menos de 5.000 millones de dólares).

${ }^{29}$ Para esto, asumimos que el valor de la elasticidad de la oferta de trabajo "macro", $\varepsilon_{k}$, se aplica también a la subpoblación beneficiaria y potencial beneficiaria. Luego, aproximamos el cambio porcentual promedio en los ingresos que implicaría el INIT como $\Delta \% Y=\overline{I N I T} / \bar{Y}$ y estimamos la cantidad de personas que se incorporarían al mercado del trabajo como:

$$
\Delta L=\varepsilon_{k} \cdot \Delta \% Y \cdot L
$$

Luego asumimos que los $\Delta L$ potenciales beneficiarios con mayor propensión a trabajar (de acuerdo a $\Phi i$ ) son los que se incorporan al mercado del trabajo. En principio, esta modificación no debería tener un sentido obvio en aumentar o disminuir el número de personas que se incorporan a la oferta de trabajo una vez se crea el INIT, ya que esto depende de qué tan cerca del margen se encontraban los potenciales beneficiarios individuales. Sin embargo, en todas nuestras simulaciones, esto hace aumentar de manera importante la aditividad, lo cual refleja que el incentivo individual a trabajar en el grupo objetivo de la política es más bajo de lo que se asume en la población total. 
Tabla 6. ANÁLISIS DE SENSIBILIDAD (EN MILLONES DE DÓLARES)

\begin{tabular}{cccccccccc}
\hline Diseño & \multicolumn{3}{c}{ Ad. micro 20h } & \multicolumn{3}{c}{ Ad. macro 45h } & \multicolumn{3}{c}{ Ad. macro 20h } \\
\hline & Adición & Gini & Costo & Adición & Gini & Costo & Adición & Gini & Costo \\
\hline 100 & 116.592 & 0,5106 & 269 & 5.354 & 0,5144 & 123 & 19.362 & 0,5139 & 147 \\
500 & 156.492 & 0,5026 & 808 & 41.019 & 0,5065 & 578 & 78.025 & 0,5058 & 668 \\
1.000 & 167.974 & 0,4952 & 1.375 & 85.600 & 0,4974 & 1.175 & 131.260 & 0,4967 & 1.301 \\
2.000 & 190.960 & 0,4815 & 2.565 & 170.705 & 0,4798 & 2.478 & 226.454 & 0,4799 & 2.655 \\
3.000 & 191.093 & 0,4726 & 3.522 & 245.728 & 0,4669 & 3.624 & 297.070 & 0,4684 & 3.766 \\
4.000 & 190.972 & 0,4655 & 4.539 & 318.781 & 0,4557 & 4.819 & 362.978 & 0,4589 & 4.909 \\
5.000 & 190.590 & 0,4609 & 5.555 & 384.467 & 0,4476 & 5.981 & 420.923 & 0,4524 & 6.026 \\
\hline
\end{tabular}

Otro punto relevante a destacar es que, al proyectar a la población efectiva la elasticidad asumida para toda la población, la aditividad efectiva es mayor que usando la elasticidad micro computada a partir de las estimaciones de la propensión individual al trabajo. De igual forma, el suponer que los trabajadores que se integrarían al mercado laboral lo harían mediante un régimen de media jornada (20 horas) también expande la aditividad en comparación a los casos que suponen incorporación a jornada completa. Una razón para explicar esto sería que el bono por cada carga, entregado por el INIT, es constante, independientemente de la cantidad de horas que la persona decida trabajar. De esta forma, esto representa un porcentaje de aumento mayor en el salario-sombra para quienes ingresan trabajando menos horas en comparación a quienes ingresan a trabajar una jornada completa, por ejemplo. Ello genera que la predicción de la probabilidad de participación, dado los ingresos adicionales por INIT, sea relativamente mayor para el primer grupo.

El análisis de los resultados anteriores sugiere que tanto los impactos en desigualdad, beneficiarios totales y nuevos empleos, así como los costos de la política debieran encontrarse dentro de los rangos presentados. Es decir, es altamente probable que algunos de los que se incorporen al mercado laboral lo hagan trabajando jornada completa (45 horas), mientras que otros lo hagan en media jornada (20 horas). Por lo que la tabla 6 nos sugeriría una suerte de intervalo de confianza para cada indicador, ya sea que asumamos elasticidad macro o micro. 


\section{CONCLUSIÓN}

Las demandas por mayor igualdad cada vez tienen más eco en la ciudadanía. Si bien existen muchas alternativas de políticas que pueden llevarse a cabo para hacer frente a este problema, ¿cómo poder saber si el costo de éstas es justificable? ¿Cuál es el costo de oportunidad de una política de este estilo? Preguntas como éstas son las que ha intentado responder este trabajo, presentando un crédito de impuestos al ingreso generado o impuesto negativo de ingreso al trabajo (el que llamamos INIT).

La mayoría de los países desarrollados no redistribuyen ingresos entre su población usando prestaciones de servicios (salud, educación o vivienda), como en Chile, sino por el mecanismo de impuestos y transferencias monetarias. Las transferencias monetarias más efectivas que se pueden proponer, aquellas que bonifican los ingresos laborales autogenerados, tienen varias condiciones deseables: primero, no distorsionan la decisión ocio-trabajo (al menos no en forma importante); segundo, promueven la inserción laboral y fomentan la formalización, toda vez que los ingresos que son bonificados son aquellos que son reportados al sistema impositivo; $y$, tercero, tienden a evitar crear dependencia del sistema por parte de las familias.

De acuerdo a nuestro análisis, con un presupuesto de 5.000 millones de dólares ${ }^{30}$ el esquema del subsidio al trabajo propuesto, que bonifica los ingresos laborales de los chilenos que tienen salarios por debajo del tramo de rentas exento y que pertenecen a los primeros siete deciles de ingresos, logra disminuir el ratio de Gini entre un 9 y un 11,8 por ciento, caída mayor en magnitud a la experimentada por la economía en el periodo 1990 y 2015, beneficiando, directa e indirectamente, a entre 9.300 .000 y 10.300 .000 personas.

${ }^{30}$ Conforme a la cuenta pública de la Tesorería General de la República del año 2016, en 2015 el Estado recaudó 44,27 billones de pesos correspondientes a 65.408 millones de pesos. Luego, un gasto de 5.000 millones de dólares corresponde al 7,7 por ciento de la recaudación total, aproximadamente. Es decir, un esfuerzo fiscal significativo. Por otro lado, según el Informe de "Política social del año 2013" (descontinuado por el actual gobierno) (http://www.ministeriodesarrollosocial.gob. cl/ipos-2013/index.php), el gasto social en ese año fue de 15.102 .824 millones de pesos o (en dólares de la época) 31.028 millones de dólares, por lo que 5.000 millones serían el 16,1 por ciento del gasto social total del país. 
Algunos elementos a tener en cuenta son que, a medida que aumenta el presupuesto, la cobertura óptima del INIT va incrementándose $\mathrm{y}$, al mismo tiempo, va cayendo la desigualdad de ingresos, aunque a tasas decrecientes. En línea con ello, destaca la relación cuadrática que surge entre el índice de Gini y el gasto asignado, hecho que permite extender el análisis presentado para cualquier punto intermedio de presupuesto, haciendo más fácil el uso del INIT como benchmark.

Se concluye que, desde la dimensión de política social pro equidad, el INIT se presenta como una alternativa sólida que la autoridad tiene a su disposición, siendo una política progresiva (al menos en un contexto estático), que va en la dirección de reducir las sustantivas brechas de ingresos que hoy aquejan a Chile.

\section{REFERENCIAS}

Agostini, C., J. Selman \& M. Perticará. 2013. "Una propuesta de crédito tributario al ingreso para Chile". Estudios Públicos 129: 49-104.

Beyer, H. 2011. “¿Qué veinte años no es nada...? Una mirada a la desigualdad de ingresos a partir de las encuestas Casen”. Estudios Públicos 121: 5-35.

Bravo, D. \& J. Valderrama. 2011. "The Impact of Income Adjustments in the Casen Survey on the Measurement of Inequality in Chile". Estudios de Economía 38 (1): 43-65.

Chetty, R. G., A. Guren, D. Manoli \& A. Weber. 2011. "Are Micro and Macro Labor Supply Elasticities Consistent? A Review of Evidence on the Intensive and Extensive Margins". The American Economic Review 101 (3): 471-475.

Chipperfield, A., P. Fleming, H. Pohlheim \& C. Fonseca. 1994. Genetic Algorithm Toolbox for Use with MATLAB. Universidad de Sheffield, Ed. http://codem. group.shef.ac.uk/public/GAToolbox_Documentation.pdf

Eissa, N. \& H. Hoynes. 2011. "Redistributional and Tax Expenditures: The Earned Income Tax Credit”. National Tax Journal 64 (2, parte 2): 689-730.

Eissa, N. \& J. Liebman. 1996. "Labor Supply Response to the Earned Income Tax Credit". Quarterly Journal of Economics 111 (2): 605-637.

Fiszbein, A., N. Schady, F. Ferreira, M. Grosh, N. Kelleher, P. Olinto y E. Skoufias. 2009. "Conditional Cash Transfers: Reducing Present and Future Poverty". Policy Research Report. The World Bank.

Heckman, J. 1979. "Sample Selection Bias as a Specification Error". Econometrica 47 (1): 153-161.

1993. "What Has Been Learned About Labor Supply in the Past Twenty Years?" The American Economic Review 83 (2): 116-121.

Kahneman, D. \& A. Tversky. 1984. "Choices, Values and Frames". American Psychologist 39 (4): 341-350. 
Larrañaga, O. \& M. Rodríguez. 2014. "Desigualdad de ingresos y pobreza en Chile: 1990 a 2013”. En Las nuevas politicas de protección social en Chile, editado por O. Larrañaga \& D. Contreras. Santiago: Uqbar Editores.

Lindert, K., E. Skoufias y J. Shapiro. 2006. "Redistributing Income to the Poor and the Rich: Public Transfers in Latin America and the Caribbean". Social Protection Discussion Paper, $n .^{\circ}$ 0605. The World Bank.

Ljungqvist, L. S. 2011. “A Labor Supply Elasticity Accord?”. The American Economic Review 101 (3): 487-491.

Meyer, B. \& D. Rosenbaum. 2001. "Welfare, the Earned Income Tax Credit, and the Labor Supply of Single Mothers". Quarterly Journal of Economics CXVI: 1063-1114.

Petridis, V., S. Kazarlis \& A. Bakirtzis. 1998. "Varying Fitness Functions in Genetic Algorithm Constrained Optimization: The Cutting Stock and Unit Commitment Problems". IEEE Transactions on Systems, Man, and Cybernetics 28 (5): 629-640.

Rau, T. 2012. "Flexibilidad de la jornada laboral en Chile: una tarea pendiente". Temas de Agenda Pública, año 7, n. ${ }^{\circ}$ 50. Centro de Políticas Públicas UC.

Reid, D. J. 1996. "Genetic Algorithms in Constrained Optimization”. Mathematical and Computer Modelling 23 (5): 87-111.

Simon, H. 1955. “A Behavioral Model of Rational Choice”. The Quarterly Journal of Economics 69 (1): 99-118.

Wah, B. \& Y.-X. Chen. 2000. "Constrained Genetic Algorithms and their Applications in Nonlinear Constrained Optimization". Proceedings of the 12th. International Conference on Tools with Artificial Intelligence. IEEE.

Yeniay, Ö. \& B. Ankara. 2005. "Penalty Function Methods for Constrained Optimization with Genetic Algorithms". Mathematical and Computational Applications 10 (1): 45-56. 


\section{APÉNDICE 1}

Resultados de las estimaciones mediante el método de Heckman

\begin{tabular}{|c|c|c|}
\hline Variables & Ecuación de salarios & Ecuación de selección \\
\hline \multirow[t]{2}{*}{ Escolaridad } & $0,0928^{* * *}$ & $0,0499^{* * *}$ \\
\hline & $(0,00238)$ & $(0,00349)$ \\
\hline \multirow[t]{2}{*}{ Edad } & & $0,129^{* * *}$ \\
\hline & & $(0,00572)$ \\
\hline \multirow[t]{2}{*}{ Edad $x$ edad } & & $-0,00158^{\star \star \star}$ \\
\hline & & $(7,06 e-05)$ \\
\hline \multirow[t]{2}{*}{ Ingresos otros integrantes } & $1,19 e-07^{* * *}$ & $-2,10 e-08^{* *}$ \\
\hline & $(1,46 e-08)$ & $(8,88 e-09)$ \\
\hline \multirow[t]{2}{*}{ Zona (urbano = 1) } & $0,0328^{* *}$ & $0,147^{\star \star \star}$ \\
\hline & $(0,0146)$ & $(0,0182)$ \\
\hline \multirow[t]{2}{*}{ Región (RM = 1) } & $0,0923^{\star * *}$ & $0,190^{* * *}$ \\
\hline & $(0,0147)$ & $(0,0256)$ \\
\hline \multirow[t]{2}{*}{ Educación madre (media = 1) } & $0,0666^{* * *}$ & $0,0816^{* *}$ \\
\hline & $(0,0213)$ & $(0,0323)$ \\
\hline \multirow[t]{2}{*}{ Educación madre (superior $=1$ ) } & $0,279^{* * *}$ & $0,162^{* * *}$ \\
\hline & $(0,0356)$ & $(0,0517)$ \\
\hline \multirow[t]{2}{*}{ Educación padre (media = 1) } & $0,101^{\star \star \star}$ & $-0,0469$ \\
\hline & $(0,0198)$ & $(0,0339)$ \\
\hline \multirow[t]{2}{*}{ Educación padre (superior = 1) } & $0,356^{\star \star *}$ & $-0,0456$ \\
\hline & $(0,0314)$ & $(0,0529)$ \\
\hline \multirow[t]{2}{*}{ Sexo (hombre = 1) } & $0,223^{* * *}$ & $1,187^{\star \star *}$ \\
\hline & $(0,0196)$ & $(0,0266)$ \\
\hline \multirow[t]{2}{*}{ Otros ocupados en hogar (si = 1) } & & $-0,258^{* * *}$ \\
\hline & & $(0,0297)$ \\
\hline \multirow[t]{2}{*}{ Integrante que ni estudie ni trabaje (si = 1) } & & 0,0237 \\
\hline & & $(0,0253)$ \\
\hline \multirow[t]{2}{*}{ Discapacitado (sí = 1) } & & $-0,592^{* \star *}$ \\
\hline & & $(0,0552)$ \\
\hline \multirow[t]{2}{*}{ Otros discapacitados en hogar (si = 1) } & & $-0,136^{\star \star *}$ \\
\hline & & $(0,0289)$ \\
\hline
\end{tabular}




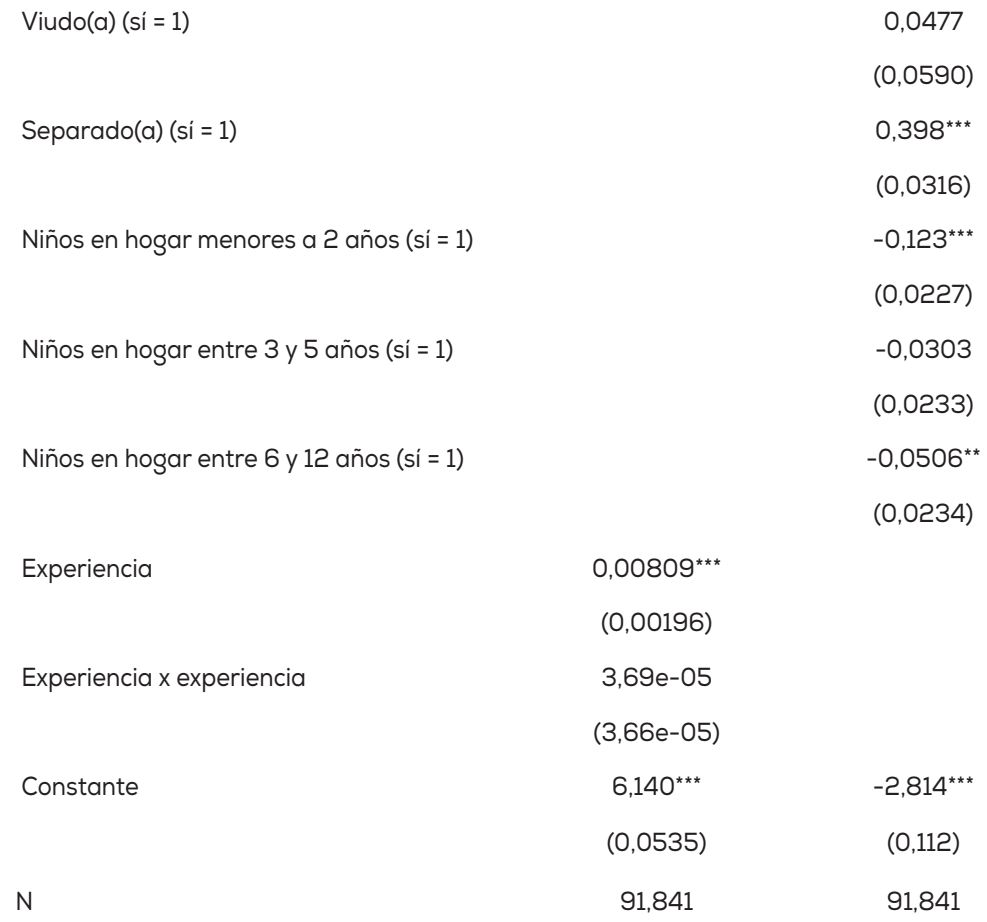

\section{APÉNDICE 2}

\section{Construcción de un estimador de la elasticidad laboral individual}

La probabilidad esperada de que un individuo trabaje dadas sus características $Z_{i}$ y el salario que podría obtener $W_{i}$ está dada por

$$
\operatorname{Prob}\left(P_{i}=1 \mid Z_{i}, W_{i}\right)=L_{i}=\Phi\left(Z_{i} \gamma+\gamma_{k} \ln \left(W_{i}\right)\right)
$$

para una población (discreta) caracterizada por una distribución $Z_{i} W_{i}$ la participación laboral esperada (como función del patrón salarial $W$ ) está dada por:

luego:

$$
\bar{L}=E[L(W) \mid Z]=\sum_{i} \Phi\left(Z_{i} \gamma+\gamma_{k} \ln \left(W_{i}\right)\right)
$$




$$
\frac{\partial \bar{L}}{\partial W_{i}}=\phi\left(Z_{i} \gamma+\gamma_{k} \ln \left(W_{i}\right)\right) \gamma_{k} \frac{1}{W_{i}}
$$

lo que implica que la elasticidad individual (extensiva) de la oferta de trabajo del individuo $i$ es:

$$
\varepsilon_{i}=\frac{W_{i}}{L_{i}} \frac{\partial L_{i}}{\partial W_{i}}=\frac{\gamma_{k}}{L_{i}} \phi\left(Z_{i} \gamma+\gamma_{k} \ln \left(W_{i}\right)\right)
$$

Es decir, para un cambio $\Delta W_{i}$ en el salario efectivo del individuo $i$ la probabilidad de que este decida trabajar cambia en:

$$
\begin{aligned}
\Delta L_{i} & =\gamma_{k} \phi\left(Z_{i} \gamma+\gamma_{k} \ln \left(W_{i}\right)\right) \frac{\Delta W_{i}}{W_{i}} \\
& \approx \gamma_{k} \phi\left(Z_{i} \gamma+\gamma_{k} \ln \left(W_{i}\right)\right) \ln \left(1+\frac{\Delta W_{i}}{W_{i}}\right) \\
& =\gamma_{k} \phi\left(Z_{i} \gamma+\gamma_{k} \ln \left(W_{i}\right)\right) \ln \left(\frac{W_{i}^{\prime}}{W_{i}}\right)
\end{aligned}
$$

Consideremos ahora un cambio de igual proporción en todos los salarios simultáneamente, tal que:

$$
\frac{\Delta W_{i}}{W_{i}} \approx \Delta \ln \left(W_{i}\right)=k
$$

entonces,

$$
\Delta L=\sum_{i} \gamma_{k} \phi\left(Z_{i} \gamma+\gamma_{k} \ln \left(W_{i}\right)\right) k
$$

Por lo que, si $\bar{W}$ es el salario promedio en la economía, entonces

$$
\begin{aligned}
\varepsilon_{L} & =\frac{\bar{W}}{L} \frac{\Delta L}{\Delta W}=\frac{\bar{W}}{\Delta W} \frac{k}{L} \sum_{i} \gamma_{k} \phi\left(Z_{i} \gamma+\gamma_{k} \ln \left(W_{i}\right)\right) \\
& =\gamma_{k} \frac{\sum_{i} \phi\left(Z_{i} \gamma+\gamma_{k} \ln \left(W_{i}\right)\right)}{\sum_{i} \Phi\left(Z_{i} \gamma+\gamma_{k} \ln \left(W_{i}\right)\right)}
\end{aligned}
$$

Luego, si conocemos un valor $\varepsilon_{L}$ de la elasticidad "macro" de la oferta de trabajo, podemos estimar la elasticidad "micro" implícita de esta forma: 


$$
\gamma_{k}=\varepsilon_{L} \frac{\sum_{i} \Phi\left(Z_{i} \gamma+\gamma_{k} \ln \left(W_{i}\right)\right)}{\sum_{i} \phi\left(Z_{i} \gamma+\gamma_{k} \ln \left(W_{i}\right)\right)}
$$

